INVESTIGACIÓN

\title{
IDENTIFICACIÓN DE VÁLVULAS Y UNIONES BRIDADAS PARA MONITOREO DE EMISIONES FUGITIVAS SEGÚN EL MÉTODO 21 DE LA EPA
}

\author{
IDENTIFICATION OF VALVES AND FLANGED JOINT FOR \\ MONITORING FUGITIVE EMISSIONS ACCORDING TO EPA METHOD \\ 21
}

MSc.Edwin Espinel Blanco ${ }^{a}$, Ing. Daniel Quintero Coronel $^{b}$, Ing. Lisneider Sanchez Ascanio ${ }^{c}$

\author{
${ }^{a}$ Universidad Francisco de Paula Santander Ocaña, Grupo de investigación GITYD \\ calle 9No 37-01 Apto 302, Ocaña, Colombia, eeespinelb@ufpso.edu.co \\ ${ }^{\mathrm{b}}$ Universidad Francisco de Paula Santander Ocaña, Grupo de investigación GITYD \\ Calle 14\# 5-15, Ocaña, Colombia, daquinteroc@ufpso.edu.co \\ ${ }^{c}$ Universidad Francisco de Paula Santander Ocaña, Grupo de investigación GITYD \\ Calle 19 \# 7-102, Ocaña, Colombia, lsancheza@ufpso.edu.co
}

Fecha de recepción: 05-03-2016

Fecha de aprobación: 11-05-2016

Resumen: Este documento presenta la identificación de válvulas y uniones bridadas de una planta para el procesamiento y transformación de hidrocarburos. La identificación se realizó con el fin de monitorear los elementos (uniones bridadas y válvulas) acorde a los lineamientos establecidos por el método 21 de la Agencia de Protección ambiental Americana para la reducción de emisiones fugitivas de compuestos orgánicos volátiles, perjudiciales para la salud y el medio ambiente. La identificación se llevó a cabo por medio de los diagramas de instrumentación y tubería. Con los datos recolectados se estableció un procedimiento de monitoreo para estos componentes, posteriormente se monitorearon una cierta cantidad de elementos, de los cuales, se obtuvo la emisión del fluido transportado en partes por millón y se planteó una posible solución para reducir las emisiones fugitivas.

Palabras clave: Compuesto orgánico volátil, Emisiones fugitivas, Método 21 de la EPA, Planta hidrocarburos.

Abstract: This paper presents the identification of valves and flanged joints of a plant for the processing and transformation of hydrocarbons. Identification was performed in order to monitor the elements (flanged joints and valves) according to the guidelines established by the method 21 of the Agency of American Environmental Protection Agency (EPA) to reduce fugitive emissions of compounds volatile organic compounds (VOC), detrimental to health and the 
environment. The identification was carried out by means of piping and instrumentation diagrams (P \& ID). With data collected proceeded to monitor a quantity of elements of which the emission of fluid transported in parts per million was obtained. A monitoring procedure for these components was established and a possible solution was proposed to reduce fugitive emissions.

The study allowed us to determine which teaching practices are not aligned to the pedagogical model of the University, which is the Social Constructivism, so it is necessary to establish an improvement plan aimed at strengthening teaching practices and that these in turn were relevant with the pedagogical model

Keywords: Volatile Organic Compound, Fugitive Emissions, EPA Method 21, Hydrocarbon Plant.

\section{INTRODUCCIÓN}

En el presente trabajo se realizó en una planta que procesa y transforma hidrocarburos con el fin de obtener productos tales como Benceno, Tolueno y Xileno. La planta está conformada por cinco unidades o plantas de proceso las cuales son U1, que realiza tres procesos, Prefraccionamiento, Unifining y Platforming, la U2 o Sulfolane, la U3 o Fraccionamiento, U4 o Hydeal, U5 o Hydrar

La U1, encargada del proceso de Prefraccionamiento en el que se realiza un corte a la nafta de rango de ebullición 130$400^{\circ} \mathrm{F}$ a $180-300^{\circ} \mathrm{F}$ para después retirar los livianos menores de 6 carbones y los pesados mayores de 9 carbones. El proceso de Unifining hace un tratamiento con $\mathrm{H} 2$ de la nafta adecuada en prefraccionamiento a través de un lecho catalítico, para retirar los contaminantes y así poder procesarla en la unidad de platforming, en éste, se realiza un reformado catalítico de la nafta para convertir los productos no aromáticos en aromáticos utilizando un catalizador a base de Platino y Renio, las reacciones que tienen lugar en este proceso son la deshidrogenación de naftenos y parafinas, y deshidrociclización de parafinas.

La U2-Sulfolane, realiza un proceso de extracción de líquido a líquido por el cual se separan los productos aromáticos de los no aromáticos utilizando el solvente sulfolane, que es fabricado por la compañía Shell. La unidad de extracción con Sulfolane está diseñada para procesar 4704 BPD de platformado con un contenido de aromáticos que puede fluctuar entre 50 y $70 \%$.

La U3-Fraccionamiento, provoca la separación de los hidrocarburos aromáticos por destilación, previo a ésta se hace un proceso de absorción que elimina las diolefinas y estabiliza los productos con el fin de evitar que por oxidación se dañe el color de los mismos.

La U4-Hydeal, esta unidad lleva acabo la hidrodealquilación térmica de tolueno (o xilenos) para la producción de benceno con un $60-65 \%$ de conversión.

La U5-Hydrar, en la cual se provoca la hidrogenación catalítica del benceno en fase líquida para la producción de ciclohexano de alta pureza, ocurre en tres reactores catalíticos en presencia de 
hidrogeno utilizando un catalizador a base de platino.

En este sentido, una línea de procesos por la cual circula un determinado fluido está conformada por uniones bridadas, válvulas, indicadores de presión, indicadores de temperatura, conexiones roscadas o soldadas, indicadores de flujo, indicadores de nivel, torres separadoras, intercambiadores de calor, tanques de almacenamiento, reactores, bombas, compresores, entre otros, los cuales permiten que un proceso pueda llevarse a cabo satisfactoriamente.

Las uniones bridadas que permiten la conexión de tramos de tuberías están conformadas por bridas, por un elemento de unión o junta (Gasket), la cual al ser apretada contra la superficie de las bridas rellena las imperfecciones entre estas provocando el sellado (Carvajalino, 2003), y por pernos, los cuales realizan el apriete de la unión.

Las bridas pueden tener diferentes configuraciones dependiendo del tipo de servicio para el cual se requieran, entre las más destacadas se encuentran las bridas de cara realzada (RF, por sus siglas en inglés), de cara plana (FF, por sus siglas en ingles) y la brida de unión de anillo (RTJ, por sus siglas en inglés). Las distintas configuraciones permiten seleccionar el tipo de junta que se debe utilizar para las bridas, las juntas pueden ser de materiales no metálicos en su totalidad, metálicos totalmente o una combinación entre materiales metálicos y no metálicos. La función del conjunto formado por bridas, junta y pernos (Unión bridada) es conectar los tramos de la tubería conteniendo el fluido que circula por la línea de procesos dada, esta capacidad de contención es vital, ya que debe permitir el paso del fluido evitando perdidas de estanqueidad.

Por otro lado, las válvulas permiten el paso o no de un fluido dado, estas se clasifican dependiendo de su actuación, es decir, si permiten el paso completo o regulado del fluido que circula por estas. En la industria de los hidrocarburos, pueden encontrarse válvulas de Globo (manual o neumática), de Bola, de mariposa, válvulas de compuerta, válvulas tipo check, entre otras. Las válvulas se conectan a la línea de proceso mediante uniones bridadas, conexiones soldadas o roscadas, las cuales deben asegurar las condiciones de estanqueidad en la línea de procesos.

Dado el tiempo de operación al que puede estar expuesto tanto uniones bridadas como válvulas en la industria de los hidrocarburos, estas pueden ocasionar emisiones fugitivas. Las emisiones provocan grandes contaminaciones al medio ambiente, daños en la salud humana y ocasionan pérdidas económicas (A-jalil, Hashim, Anwar, \& Sapia, 2012) relacionadas con el desperdicio de hidrocarburos importantes en la industria. Dentro de los contaminantes que provocan las emisiones se encuentran gases de efecto invernadero y emisiones productos de la combustión mezclados con vapores de VOC (Mallol, Sole, Asher, Clayton, Stein, $\&$ Soto, 2000).

En el caso de las emisiones de VOC, estudios han determinado que varios de estos compuestos provocan efectos cancerígenos, mientras que otros pueden afectar el sistema inmunológico, el sistema nervioso central (cerebro), el hígado y los riñones (United States Envioronmental Protection Agency (EPA), 2207). 
La EPA ha estimado que aproximadamente 70367 toneladas por año de VOC y 9357 toneladas por año de contaminantes volátiles peligrosos para el aire (VHAP, por sus siglas en inglés) son emitidas por equipos que intervienen en el procesamiento y transformación de hidrocarburos (Chang-Fu, Hashmonay, \& Chang, 2014). Con el fin de evaluar el impacto de compuestos orgánicos volátiles liberados en el medio ambiente y la salud pública y para desarrollar un control efectivo las estrategias, las estimaciones de emisiones específicas de la fuente son necesarias (Mimi \& Hassim, 2012).

Las emisiones fugitivas en las válvulas, y uniones bridadas contribuyen a más de un millón de toneladas métricas de emisiones por año. La eliminación o reducción de las fugas en la industria de la industria podrían ahorrar muchos millones de dólares y prolongar los recursos escasos. Las naciones industrializadas del mundo están incrementando proporcionalmente el énfasis en el control de compuestos orgánicos volátiles y contaminantes peligrosos del aire que se emiten a la atmósfera (Onat, 2008). Los Sistemas de sellado es el factor a tratar para la reducción de emisiones (Bramsiepe, Pansegrau, \& Schembecker, 2010). El sistema de sellado se ve afectado por la influencia aleatoria de las vibraciones, cambios de temperatura y los procesos de puesta en marcha o parada que se producen en las plantas que operan (Mathan \& Siva Prasad, 2012). La consideración de estas vibraciones es importante en el diseño de juntas a prueba de fugas. Las normas actuales en los códigos de fabricación están orientadas a reducir la probabilidad, de grietas producidas por fatiga en las bridas (United States Envioronmental Protection Agency (EPA), 2207).

\section{METODOLOGÍA}

Se identificaron las uniones bridadas y las válvulas para una planta de procesamiento y transformación de hidrocarburos.

La metodología se desarrolló en tres etapas, en la primera se muestra la forma como se identificaron los elementos ya mencionados, la segunda etapa muestra los puntos donde son monitoreados los componentes identificados acorde a los lineamientos del método 21 de la EPA, por último, la tercera etapa muestra una alternativa propuesta para la reducción de emisiones fugitivas.

\section{Etapa I}

La identificación de las uniones bridadas y de las válvulas se realizó por medio de P\&ID de la siguiente manera:

Con el P\&ID impreso de una unidad determinada y siguiendo el flujo de la línea de procesos (Figura 1), se tomaron datos como: Tipo de componente, Fluido transportado, Rating, Material, Temperatura y Presión.

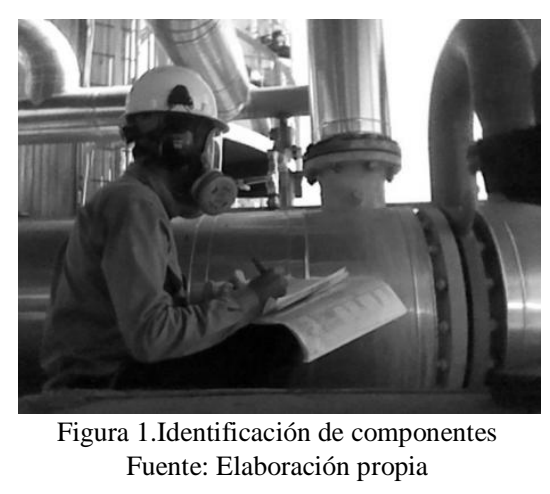

Para el caso de las uniones bridadas la toma de datos consistió en determinar qué 
tipo de bridas y junta poseía la unión bridada (Figura 2), en el caso de bridas RF, el material de la junta se determina dependiendo del color de la misma.

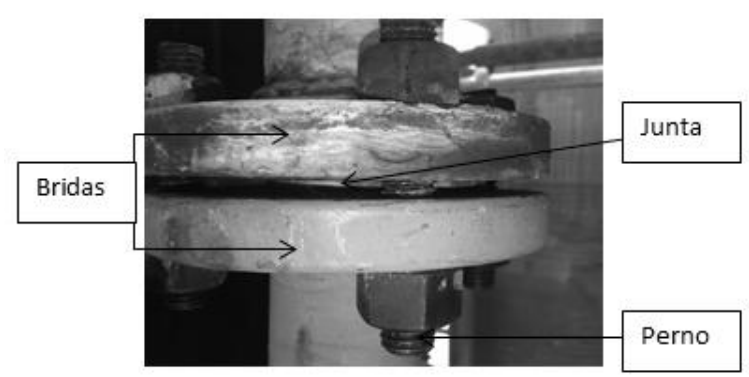

Figura 2. Unión bridada Fuente: Elaboración propia

La nomenclatura utilizada en la identificación fue la siguiente:

- F: Indica una unión bridada.

- Fr: Indica una unión bridada, pero con las bridas roscadas.

- V: Indica una válvula con conexión bridada a la tubería

- Vr: Indica una válvula con conexión roscada a la tubería.

- Vw: Indica una válvula con conexión soldada a la tubería.

Para el caso de las válvulas, además de los datos mencionados inicialmente en la etapa I, se identificó que tipo de conexión presentaba con la tubería (Figura 3).

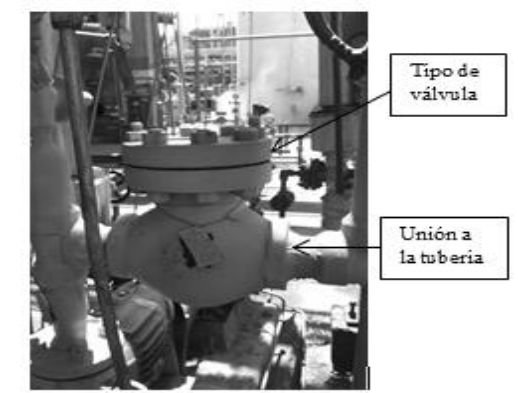

Figura 3.Forma de identificación de las válvulas Fuente: Elaboración propia

Etapa II

Según la EPA, Una fuente de emisiones fugitivas es cualquier dispositivo o elemento que debido a fallas en el material de sellado provoca que el producto transportado escape (United States Envioronmental Protection Agency (EPA), 2207).

El método 21 de la EPA usa un instrumento portátil para detectar fugas de VOC de fuentes individuales, el tipo del instrumento debe ser capaz de detectar las emisiones de los productos que están siendo transportados en la línea de procesos de la planta. El instrumento debe ser calibrado según el producto que va a ser medido. El método establece una definición de fuga, es decir, un valor en partes por millón (ppm) en la cual se considere que un componente está fugando, esta definición de fuga se establece teniendo en cuenta el tipo de componente, la clase de fluido que transporta y las regulaciones ambientales dentro de las cuales se encuentra inmerso (Chemical Manufacturing Industry Regulations, 1998).

Las definiciones de fuga están establecidas por componentes (Tabla 1).

Tabla 1. Definiciones de fugas por componentes

\begin{tabular}{|c|c|c|c|}
\hline \multirow[b]{2}{*}{ Equipo } & \multicolumn{3}{|c|}{ Definición de fuga } \\
\hline & $\begin{array}{c}40 \text { CFR } 60 \\
\text { Subparts } \\
\text { VVa } \\
\text { (SOCMI) \& } \\
\text { GGGa } \\
\text { (Refinery) }\end{array}$ & $\begin{array}{c}40 \text { CFR } 61 \\
\text { Subparts F, } \\
J \\
\text { and V and } \\
40 \text { CFR } 63 \\
\text { Subpart } \\
\text { HH - PVC, } \\
\text { Benzene, } \\
\text { and Oil \& } \\
\text { Natural } \\
\text { Gas }\end{array}$ & $\begin{array}{c}40 \text { CFR } 60 \\
\text { Subparts } \\
\text { VV } \\
\text { (SOCMI), } \\
\text { GGG } \\
\text { (Refinery) \& } \\
\text { KKK (Gas } \\
\text { Processing } \\
\text { Plants) }\end{array}$ \\
\hline $\begin{array}{lr}\text { Válvulas } & \text { de } \\
\text { gas } & \text { o } \\
\text { líquido } & \end{array}$ & 500 ppm & 10000 ppm & 10000 ppm \\
\hline
\end{tabular}




\begin{tabular}{|c|c|c|c|}
\hline ligero & & & \\
\hline $\begin{array}{l}\text { Válvulas de } \\
\text { líquido } \\
\text { pesado }\end{array}$ & 10000 ppm & 10000 ppm & 10000 ppm \\
\hline $\begin{array}{l}\text { Bombas } \\
\text { con líquido } \\
\text { ligero }\end{array}$ & 2000 ppm & 10000 ppm & 10000 ppm \\
\hline $\begin{array}{l}\text { Bombas } \\
\text { con líquido } \\
\text { pesado }\end{array}$ & 10000 ppm & 10000 ppm & 10000 ppm \\
\hline $\begin{array}{l}\text { Válvulas de } \\
\text { control }\end{array}$ & 500 ppm & 500 ppm & 500 ppm \\
\hline $\begin{array}{l}\text { Dispositivos } \\
\text { de alivio de } \\
\text { presión }\end{array}$ & 500 ppm & 500 ppm & 500 ppm \\
\hline $\begin{array}{l}\text { Conexiones } \\
\text { (bridas y } \\
\text { uniones } \\
\text { roscadas }\end{array}$ & 500 ppm & 500 ppm & 500 ppm \\
\hline
\end{tabular}

Fuente: Elaboración propia

En este caso, se estableció una definición de fuga de 10 ppm, esto para cualquier servicio. Las definiciones de fuga mostradas la tabla anterior son muy elevadas dadas las características de los fluidos transportados en la planta, por tal razón se optó por una definición de fuga mucho menor.

Para este trabajo, el monitoreo de componentes se llevó acabo con el analizador de vapores tóxicos TVA 1000B (Figura 4), el cual cumple con los requisitos exigidos por la EPA. Este instrumento mide vapores tóxicos usando dos tecnologías diferentes, la primera es FID (Flame Ionization Detection) y PID (Photo Ionization Detection).

El detector de flama ionización (FID) mide compuestos orgánicos utilizando una flama producida por la combustión de hidrogeno y aire. Los compuestos en la muestra son introducidos en la zona de detección, en esta zona los iones son producidos mediante la reacción química(1) (Thermo Environmental Instruments, Inc. , 2001):

$$
\mathrm{RH}+\mathrm{O} \rightarrow \mathrm{RHO}^{+}+e^{-} \rightarrow \mathrm{H}_{2} \mathrm{O}+\mathrm{CO}_{2}
$$

Donde $\mathrm{R}$ indica el compuesto de carbono Un electrodo colector con un voltaje de polarización está localizado dentro de la cámara del detector. Como los iones migran hacia el colector, una corriente es producida, ésta es directamente proporcional a la concentración de hidrocarburos producidos en la flam.

La detección por medio de fotoionización (PID) consiste de una lámpara de luz ultravioleta (UV) de una energía específica y una cámara de ionización. Los compuestos pasan a través de la cámara siendo excitados e ionizados por los fotones de UV de acuerdo a la siguiente ecuación (Thermo Environmental Instruments, Inc. , 2001):

$$
R+h v \rightarrow R++e-
$$

Donde R indica el compuesto de carbono. Estos iones son atraídos por un electrodo colector, produciendo una corriente proporcional a la concentración del compuesto.

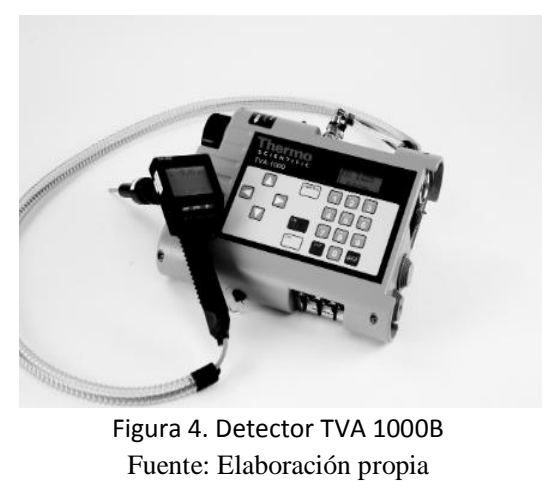


Con el detector TVA 1000B se estableció en qué puntos de las uniones bridadas y de las distintas válvulas se debe posicionar la sonda detectora del instrumento para poder tomar los datos de emisiones.

Para el caso de Uniones bridadas, la sonda del instrumento debe ser posicionada como se muestra en la figura 5, y una vez hecho esto se debe desplazar a lo largo de toda la periferia de la unión (entre la brida y la junta) para obtener una lectura adecuada con el detector de emisiones.

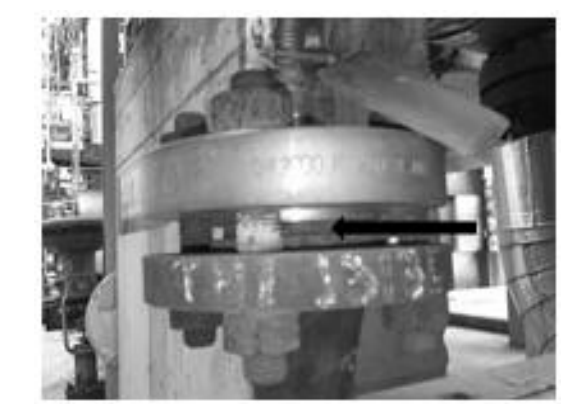

Figura 5. Punto de posicionamiento para una unión bridada. Fuente: Elaboración propia

Para el caso de las válvulas los puntos de posición se establecieron como todos aquellos donde se podía presentar una emisión (Figura 6, 7 y 8).

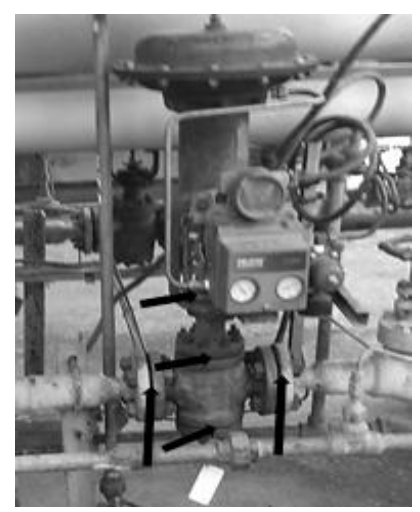

Figura 6. Punto de posicionamiento para una válvula tipo Globo con accionamiento neumático

Fuente: Elaboración propia

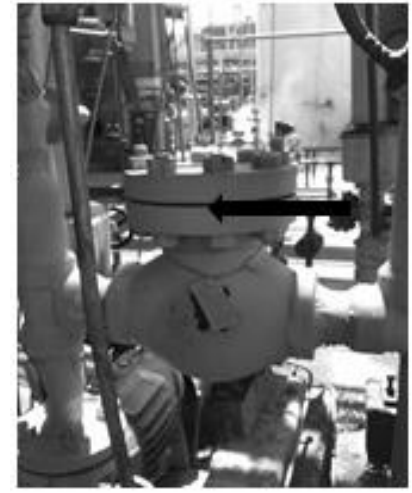

Figura 7. Punto de posicionamiento para una válvula tipo Check. Fuente: Elaboración propia

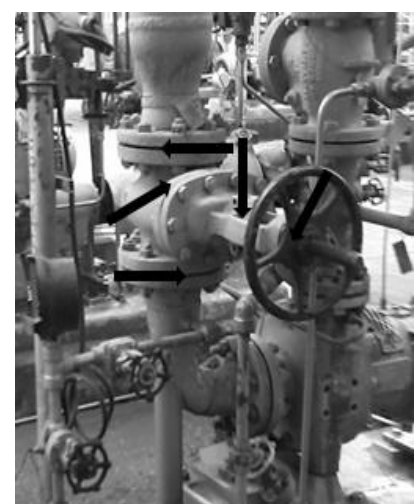

Figura 8. Punto de posicionamiento para una válvula tipo Globo con accionamiento manual Fuente: Elaboración propia

Para el caso de válvulas de compuerta los puntos para el monitoreo de emisiones son los mismos que para válvulas de globo con accionamiento manual.

\section{Etapa III}

En esta etapa se planteó un procedimiento para la reparación de aquellos componentes que una vez realizado el monitoreo de emisiones presentaran fugas acorde a la definición de fuga establecida.

La reparación del componente consiste en llevar al equipo a aquella condición en la cual el nivel de emisión esté por debajo de la definición de fuga aplicada. Para poder llevar un equipo a su condición actual, se 
deben tener claros los procedimientos de reparación, estos han de seguirse al pie de la letra, para asegurar que el componente quede funcionando correctamente.

Un primer intento de reparación debe hacerse una vez detectada la fuga y dentro de los primeros cinco días, si la fuga no puede ser reparada dentro de este tiempo deberá ser reparada dentro de los quince días siguientes, cuando los tiempos de reparación no pueden ser llevados dentro de ese intervalo, se debe proporcionar una fecha para la cual se considere que la fuga podrá ser reparada.

Si la reparación de cualquier componente es técnicamente inviable sin un proceso de shutdown, el componente puede ser localizado en una lista de reparaciones atrasadas, con el número de identificación del equipo y una explicación de porqué el equipo no puede ser reparado, una fecha estimada para la reparación del equipo debe ser proporcionada en el registro de datos.

La primera fase de reparación normalmente se reduce a operaciones de apriete, ya sea de los tornillos de la caja de empaquetaduras, de las bridas, de las uniones roscadas. Normalmente son reparaciones exitosas en la reducción de emisiones por debajo de la definición de fuga aplicada. Un apriete excesivo puede dañar una junta o empaquetadura $y$ aumentar aún más las fugas. Lo mismo puede ocurrir si se realiza un apriete desigual a los pernos de la brida. También, apretar los pernos en el orden equivocado puede crear tensiones en las juntas que desemboquen en fugas mayores.

Se recomendó seguir el siguiente procedimiento para los primeros intentos de reparación:

- Apretar el primer perno una media vuelta. El apriete del perno debe estar de acuerdo con las secuencias de aprietes establecidas en el el código ASME PCC- 1 directriz para atornillado con bridas 2010 [13].

- Cuando hay bridas de más de cuatro pernos, se debe seguir apretando los pernos de manera similar, cruzando la brida para proporcionar tensiones iguales alrededor de la junta.

- Una vez que todos los pernos han sido apretados media vuelta, se debe medir la unión bridada con el detector de emisiones para determinar si la fuga fue contrarrestada, es decir para constatar que el equipo quedó por debajo de la definición de fuga aplicada.

- Cuando el nivel de fuga es aceptable, la reparación del componente ha terminado. De no ser así, se deben apretar los pernos otra media vuelta siguiendo las misma secuencia de apriete inicial.

- Medir nuevamente las emisiones y repita los pasos anteriores hasta que el nivel de fuga haya disminuido hasta un valor aceptable. Cuando la fuga persiste y los pernos no pueden ser apretados más, se debe proceder a hacer un cambio completo del componente o solo de su material de estanqueidad.

Por otro lado, cuando se presentan fugas en las válvulas, estas tienen lugar en el prensa empaque del vástago. Cuando se mide con un detector de emisiones y el valor medido está por encima de la definición de fuga aplicada, se considera 
que el componente está fugando, el primer paso a aplicar cuando se presenta esta situación es reapretar las tuercas del prensa empaque hasta aproximadamente media vuelta, una vez hecho esto se debe medir nuevamente con el detector para asegurarse que la fuga ha sido erradicada. Si la fuga persiste, se debe proceder al reempacado del vástago, éste debe realizarse cuando se haya interrumpido el flujo del fluido por la válvula. Cuando la válvula no puede ser monitoreada, o no se puede reparar por razones de seguridad o de cualquier otro tipo, se debe especificar una fecha tentativa para su reparación

Debido a la continua operación en las plantas de procesamiento y transformación de hidrocarburos, el único método de reparación que puede realizarse en campo para una válvula de compuerta y de globo que son las que principalmente se usan, es el reapriete del prensa empaque, esto dado que para poder realizar el cambio de la válvula o el re-empacado de su vástago, se debe detener el fluido transportado, lo que no es conveniente para la planta, ya que parar el flujo de una determinada línea ocasionaría que se interfieran los demás flujos, por lo tanto el re-empacado o cambio de una válvula solo debe hacerse en las paradas de planta.

\section{RESULTADOS}

\section{Identificación de componentes}

En total se identificaron 3.686 válvulas y 3.631 uniones bridadas (Tabla 2)

Tabla 2. Componentes identificados

\begin{tabular}{|c|c|c|}
\hline Unidad & $\begin{array}{c}\text { Cantidad de } \\
\text { válvulas }\end{array}$ & $\begin{array}{c}\text { Cantidad de uniones } \\
\text { bridadas }\end{array}$ \\
\hline 1 & 1630 & 1880 \\
\hline
\end{tabular}

\begin{tabular}{|c|c|c|}
\hline 2 & 654 & 610 \\
\hline 3 & 735 & 689 \\
\hline 4 & 667 & 452 \\
\hline Total & 3686 & 3631 \\
\hline
\end{tabular}

Se creó una base de datos con todos los componentes identificados (Tabla 3), en esta se registró el fluido que circula por la línea, el tipo de componente, el P\&ID donde se encuentra, el Tag de la Línea, el lazo de corrosión, la ubicación del componente (a qué otros componentes pertenece, una bomba, un compresor), material del componente, rating, material del empaque, entre otros.

Tabla 3. Extracto base de datos

\begin{tabular}{|c|c|c|c|c|}
\hline SERVICIO & JUNTA & TAG de la Línea & $\begin{array}{c}\text { LAZO DE } \\
\text { CORROSIÓN }\end{array}$ & P\&ID \\
\hline H.C LIQUID & F1 & 3"15.2 & LC1600-01 & EF-366 \\
\hline H.C LIQUID & V1 & $3 " 15.2$ & LC1600-01 & EF-366 \\
\hline H.C LIQUID & F2 & $3 " 15.2$ & LC1600-01 & EF-366 \\
\hline H.C LIQUID & V2 & 3"15.2 & LC1600-01 & EF-366 \\
\hline H.C LIQUID & F3 & $3 " 15.2$ & LC1600-01 & EF-366 \\
\hline H.C LIQUID & F4 & 3"15.2 & LC1600-01 & EF-366 \\
\hline H.C LIQUID & V3 & 3"15.2 & LC1600-01 & EF-366 \\
\hline H.C LIQUID & F5 & $3 " 15.2$ & LC1600-01 & EF-366 \\
\hline H.C LIQUID & V4 & $3 " 15.2$ & LC1600-01 & EF-366 \\
\hline H.C LIQUID & F6 & 3"15.2 & LC1600-01 & EF-366 \\
\hline H.C LIQUID & V5 & 3"15.2 & LC1600-01 & EF-366 \\
\hline H.C LIQUID & F7 & 3"15.2 & LC1600-01 & EF-366 \\
\hline H.C LIQUID & F8 & $3 " 15.2$ & LC1600-01 & EF-366 \\
\hline H.C LIQUID & V6 & $3 " 15.2$ & LC1600-01 & EF-366 \\
\hline H.C LIQUID & F9 & 3"15.2 & LC1600-01 & EF-366 \\
\hline
\end{tabular}




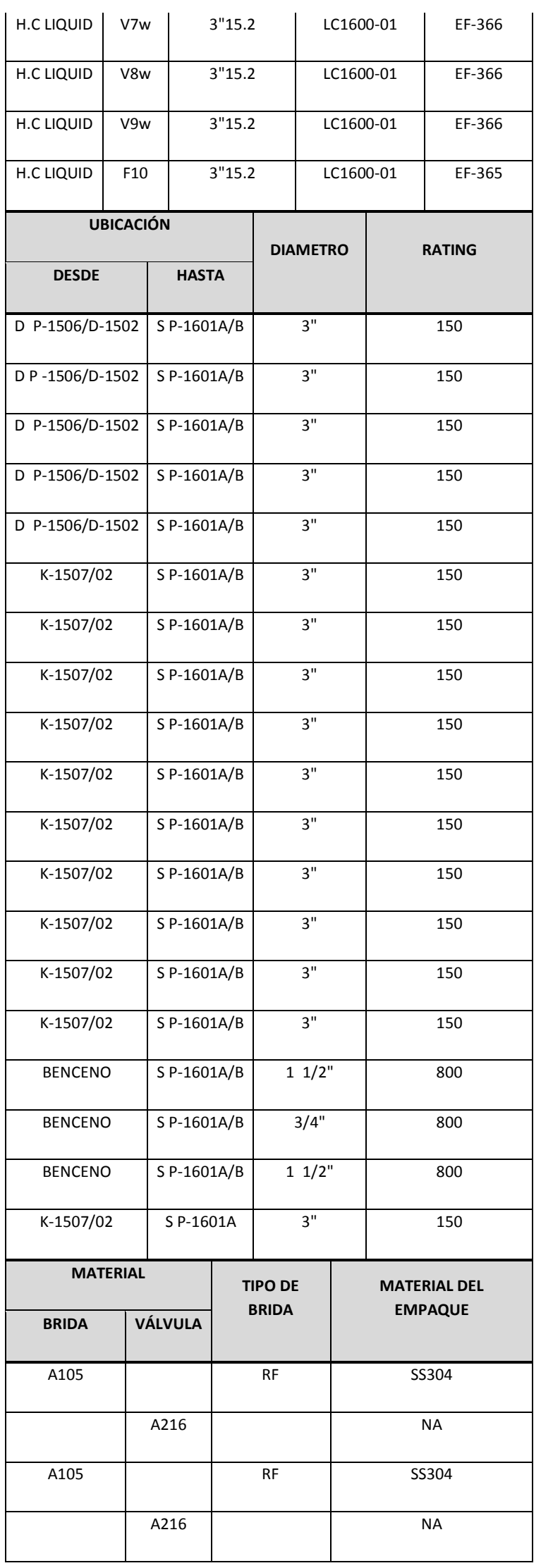

\begin{tabular}{|c|c|c|c|}
\hline A105 & & $\mathrm{RF}$ & SS304 \\
\hline \multirow[t]{2}{*}{ A105 } & & $\mathrm{RF}$ & SS304 \\
\hline & A216 & & NA \\
\hline \multirow[t]{2}{*}{ A105 } & & $\mathrm{RF}$ & SS304 \\
\hline & A216 & & NA \\
\hline \multirow[t]{2}{*}{ A105 } & & $\mathrm{RF}$ & SS304 \\
\hline & A216 & & NA \\
\hline A105 & & $\mathrm{RF}$ & SS304 \\
\hline \multirow[t]{2}{*}{ A105 } & & $\mathrm{RF}$ & SS304 \\
\hline & A216 & & NA \\
\hline \multirow[t]{4}{*}{ A105 } & & $\mathrm{RF}$ & SS304 \\
\hline & A216 & & NA \\
\hline & A216 & & NA \\
\hline & A216 & & NA \\
\hline A105 & & $\mathrm{RF}$ & SS304 \\
\hline
\end{tabular}

\section{Registro de emisiones}

Se tomaron datos de algunos componentes con el fin de conocer el estado de emisión que presentaba (Tabla 4), se registraron estos datos y se procedió a realizar los primeros intentos de reparación que consiste en operaciones de apriete de los pernos tanto de las uniones bridadas como de válvulas.

Tabla 4. Datos de emisiones de componentes

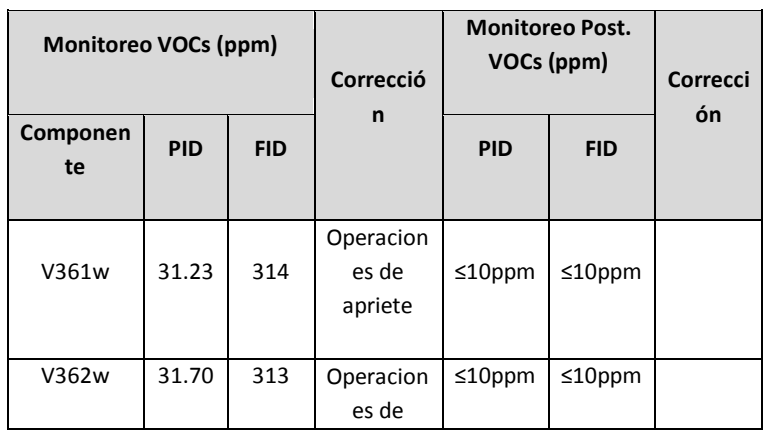




\begin{tabular}{|c|c|c|c|c|c|c|}
\hline & & & apriete & & & \\
\hline V364 & 34.40 & 57.61 & $\begin{array}{l}\text { Operacion } \\
\text { es de } \\
\text { apriete }\end{array}$ & $\leq 10 \mathrm{ppm}$ & $\begin{array}{c}\geq 10 p p \\
m\end{array}$ & $\begin{array}{c}\text { Reempa } \\
\text { cado de } \\
\text { la } \\
\text { válvula }\end{array}$ \\
\hline V365 & 30.04 & 20.71 & $\begin{array}{l}\text { Operacion } \\
\text { es de } \\
\text { apriete }\end{array}$ & $\leq 10 \mathrm{ppm}$ & $\begin{array}{c}\geq 10 p p \\
m\end{array}$ & $\begin{array}{c}\text { Reempa } \\
\text { cado de } \\
\text { la } \\
\text { válvula }\end{array}$ \\
\hline V366 & 289 & 38892 & $\begin{array}{l}\text { Operacion } \\
\text { es de } \\
\text { apriete }\end{array}$ & $\leq 10 \mathrm{ppm}$ & $\begin{array}{c}\geq 10 p p \\
m\end{array}$ & $\begin{array}{c}\text { Reempa } \\
\text { cado de } \\
\text { la } \\
\text { válvula }\end{array}$ \\
\hline V380 & 28.27 & 12.08 & $\begin{array}{l}\text { Operacion } \\
\text { es de } \\
\text { apriete }\end{array}$ & $\leq 10 \mathrm{ppm}$ & $\begin{array}{c}\geq 10 p p \\
m\end{array}$ & $\begin{array}{l}\text { Reempa } \\
\text { cado de } \\
\quad \text { la } \\
\text { válvula }\end{array}$ \\
\hline V381w & 30.39 & 22.05 & $\begin{array}{c}\text { Operacion } \\
\text { es de } \\
\text { apriete }\end{array}$ & $\leq 10 \mathrm{ppm}$ & $\leq 10 \mathrm{ppm}$ & \\
\hline V382w & 31.44 & 49.28 & $\begin{array}{c}\text { Operacion } \\
\text { es de } \\
\text { apriete }\end{array}$ & $\leq 10 \mathrm{ppm}$ & $\leq 10 \mathrm{ppm}$ & \\
\hline V384 & 30.52 & 38.26 & \begin{tabular}{|c|} 
Operacion \\
es de \\
apriete
\end{tabular} & $\leq 10 \mathrm{ppm}$ & $\leq 10 \mathrm{ppm}$ & \\
\hline
\end{tabular}

\section{CONCLUSIONES}

Se lograron identificar las uniones bridadas y las válvulas de la planta de procesamiento y transformación de hidrocarburos.

Se creó una base de datos completa con el fin de saber qué componente pertenece a determinada línea, facilitando las operaciones de reparación de los mismos, ya que muestra todos los datos necesarios de los componentes.

Se estableció la forma de monitorear los elementos acorde al método 21 de la EPA. Se estableció y se recomendó seguir un procedimiento para las operaciones de reparación de los componentes.
Los componentes monitoreados presentaron niveles de emisión por encima de la definición de fuga establecida por lo que fue necesario intervenirlos. Los componentes a los cuales se les realizaron las primeras operaciones de reparación y seguían presentando fugas, se llevaron a la segunda etapa de reparación que consiste en el reempacado de los componentes.

Las reparaciones de apriete y reempacado en forma general fueron satisfactorias, aunque algunos componentes que seguían presentando fugas necesariamente debieron ser cambiados.

\section{BIBLIOGRAFÍA}

A-jalil, Hashim, M., Anwar, J., \& Sapia, N. (2012). Fugitive Emission Reduction Using Mixed Integer Linear. Energy Procedia , 14, 1870-1876.

Bramsiepe, C., Pansegrau, L., \& Schembecker, G. (2010). A model to predict fugitive VOC emissions from liquid charged flange joints with graphite gaskets. Chemical and Engineering Journal , 1385-1394.

Carvajalino, C. (2003). Juntas Industriales. Rio de Janeiro.

Chang-Fu, W., Hashmonay, R., \& Chang, S. (2014). Measurement of fugitive volatile organic compound emissions from a petrochemical tank farm using open-path Fourier transform infrared spectrometry. Atmospheric Environment, 335-342. 
Chemical Manufacturing Industry Regulations. (1998). Inspection Manual: Federal Equipament Leak Regulations for the Chemical Manufacturing Industry. Envioremental Protection Agency.

Mallol, J., Sole, D., Asher, I., Clayton, T., Stein, R., \& Soto, M. (2000). Prevalence of Asthma Symtoms in Latin America: the International Study of Asthma and Allergies in Childhood (ISAAC). Pediatric Pullmonology, 30 (6), 39-44.

Mathan, G., \& Siva Prasad, N. (2012). Study of dynamic response of piping system with gasketed flanged joints using finite element analysis. International Journal of Pressure Vessels and Piping, 28-32.

Mimi, H., \& Hassim, M. H. (2012). Fugitive emissions in chemical processes: The assessment and prevention based on inherent and add-on approaches. Journal of Loss Prevention in the Process Industries, 820-829.

Onat, A. (2008). the effects of sealing materials on elimination of fugitiva emissions. Materials \& Design, 533-538.

Thermo Environmental Instruments, Inc. . (2001). www.geotechenv.com. Recuperado el $14 \mathrm{de}$ Marzo de 2014

United States Envioronmental Protection Agency (EPA). (2207). Leak Detection and Repair. Pennsylvania. 